\title{
Incoherent interlayer electron hopping as a possible reason for enhanced magnetic quantum oscillations in the mixed state of a layered organic superconductor
}

\author{
V.M. Gvozdikov ${ }^{1,2}$ and J. Wosnitza ${ }^{3,4}$ \\ ${ }^{1}$ Kharkov National University, 61077, Kharkov, Ukraine \\ ${ }^{2}$ Max-Planck-Institut für Physik Komplexer Systeme, D-01187 Dresden, Germany \\ ${ }^{3}$ Institut für Festkörperphysik, Technische Universität Dresden, D-01062 Dresden, Germany \\ ${ }^{4}$ Forschungszentrum Rossendorf, Hochfeld-Magnetolabor Dresden (HLD), D-01314 Dresden, Germany \\ E-mail: Vladimir.M.Gvozdikov@univer.kharkov.ua
}

Received July 11, 2005

\begin{abstract}
We present a theory which is able to explain enhanced magnetic quantum-oscillation amplitudes in the superconducting state of a layered organic metal with incoherent electronic transport across the layers. The incoherence acts through the deformation of the layer-stacking factor which becomes complex and decreases the total scattering rate in the mixed state. This novel mechanism restores the coherence by establishing a long-range order across the layers and can compensate the usual decrease of the Dingle factor below the upper critical magnetic field caused by the intralayer scattering.
\end{abstract}

PACS: 71.18.+y, 72.15.Gd, 74.70.Kn

Keywords: magnetic quantum oscillations, incoherence, layered organic superconductor

\section{Introduction}

It is well known that magnetic field and superconductivity are antagonists in the sense that a superconductor tend to expel magnetic field from its interior: completely in the Meissner state and partially in the Abrikosov vortex state. Because of that, a foundation in 1976 the coexistence of magnetic quantum oscillations and superconductivity in layered dichalcogenides of transient metals [1] was at first considered just as a strange experimental fact. Only a decade and a half later was it recognized that the magnetic quantum oscillations in layered superconductors is not an artefact but a general and complex phenomenon waiting for systematic studies. A history of these studies and a survey of the related theoretical ideas can be found in the review paper [2].

Since the first observation of the de Haas - van Alphen (dHvA) oscillations in the mixed state of layered superconductors it has been firmly established in numerous experiments that these oscillations are always damped below the upper critical field $B_{c 2}$. This damping was explained by several mechanisms reviewed in [2]. The recent finding that both the dHvA and the Shubnikov-de Haas ( $\mathrm{SdH})$ amplitudes are enhanced in the mixed state of the layered organic superconductor $\beta "$-(BEDT-TTF) ${ }_{2} \mathrm{SF}_{5} \mathrm{CH}_{2} \mathrm{CF}_{2} \mathrm{SO}_{3}$ ( $\beta^{\prime \prime}$ salt in the following) [3] was a real surprise because it is in sharp contrast to all experiments and theories known so far [2]. Up to now it has been assumed that the quasiparticle scattering by the «vortex matter» is the main mechanism of the damping which acts through additional Dingle-like factor $R_{S}=$ $=\exp \left(-2 \pi / \Omega \tau_{\mathrm{sc}}\right)$. Here $\Omega$ is the cyclotron frequency, $\tau_{\mathrm{sc}}$ is the scattering time. The Landau-level broadening, $\hbar / \tau_{\mathrm{sc}}$, is a sum of the two terms: $\hbar / \tau_{\mathrm{sc} 1}=$ $=\Delta^{2}(\pi / \mu \hbar \Omega)^{1 / 2}$, which is due to the intralayer scattering at vortices [4-6], and $\hbar / \tau_{\mathrm{sc} 2}=\beta \Delta^{2} / \mu \Omega \tau_{0}$, which takes account of the additional cooperative scattering effect by impurities and vortices within the layers [7] ( $\mu$ is the chemical potential, $\Delta$ is the 
superconducting order parameter which just below the $B_{c 2}$ is less than the Landau-level separation $\Delta<<\hbar \Omega$, and $\beta \simeq 1$ ). It is important to note that all theories so far were developed for the $2 \mathrm{D}$ superconductors. On the other hand, it was shown yet in 1984 [8] that electron hopping across the layers strongly affects oscillations through a special layer-stacking factor which together with the standard Dingle, spin, and temperature factors modulates the dHvA amplitudes. The case of the $\mathrm{SdH}$ oscillations is more complex because of the incoherence and localization associated with the electron hopping between the layers [9].

In the 2D superconductors the additional Dinglelike factor $R_{S}$ is due to the intralayer scattering only. It describes the extra damping of the dHvA and $\mathrm{SdH}$ amplitudes so that the enhancement of the oscillations in the mixed state of the $\beta^{\prime \prime}$ is absolutely unclear. To understand the anomalous enhancement of the magnetic quantum oscillations in the $\beta$ " one can first rise a question what is unusual in this superconductor compared to conventional layered superconductors. The answer is that the $\beta^{\prime \prime}$ is the only known superconductor which (i) displays enhanced magnetic quantum-oscillation amplitudes in the mixed state both in the dHvA and the SdH signals and (ii) exhibits an incoherent electronic transport across the layers [10]. The incoherence means that the electronic properties of layered quasi-2D metals can not be described within the usual fundamental concept of an anisotropic 3D Fermi surface (FS) [11]. The $\beta "$ salt belongs to organic conductors of the type (BEDT-TTF) $)_{2} \mathrm{X}$, where BEDT-TTF stands for bisethylenedithio-tetra-thiafulvalene and $\mathrm{X}$ for a monovalent anion. This class of materials displays a number of unique properties such as an unconventional electronic interlayer transport, clear deviations of their magnetic quantum oscillations [12] from the standard three-dimensional (3D) Lifshitz-Kosevich theory [13], and puzzling enhancement of the magnetic quantum oscillations in the superconducting mixed state. This enhancement cannot be explained by the $2 \mathrm{D}$ theories.

In this paper we consider a new mechanism for the $\mathrm{SdH}$ and dHvA amplitude modulation in the mixed state. This mechanism goes beyond the 2D consideration and takes account of the incoherent interlayer hopping. The physical picture behind this mechanism is as follows. The incoherence, or disorder in direction perpendicular to the layers, hampers the electron hopping between neighboring layers. This enhances the scattering at impurities within the layers since electrons on the Landau orbit interact with the same impurities many times before a hopping to the neighboring layer. In the superconducting state a long- range order establishes across the layers which allows quasiparticles to escape the intralayer multiple scattering by the Josephson tunnelling between the layers. This reduces the scattering rate by impurities and enhances the Dingle factor in the superconducting state. For the $\beta$ " salt this effect most likely plays a dominant role. In the coherent case, there is no interlayer scattering and electrons (quasiparticles) can move freely across the layers with the fixed momentum both in the normal and superconducting states which render the above mechanism much less effective. Numerically, this effect is described by the layer-stacking factor [Eq. (3)] which itself contains a Dingle-like exponent in the case of incoherent interlayer hopping [8]. Superconductivity restores the coherence across the layers by renormalizing the hopping integrals [14]. This reduces the interlayer scattering and enhances the oscillation amplitudes.

Anomalous dHvA oscillations have also been observed in $\mathrm{YNi}_{2} \mathrm{~B}_{2} \mathrm{C}[15,16]$. These oscillations persist down to the surprisingly low field $0.2 B_{c 2}$ [16]. A Landau-quantization scheme for fields well below $B_{c 2}$ in the periodic vortex-lattice state was developed in [17] and for a model with an exponential decrease of the pairing matrix elements in [7]. The observed recovery of the dHvA amplitudes for $B<B_{c 2}$ in the borocarbide $\mathrm{YNi}_{2} \mathrm{~B}_{2} \mathrm{C}$ was explained by the enhancement of the special vortex-lattice factor depending on the Landau bands which become narrower when the vortex lattice grows thinner [7].

The anomalous magnetic quantum oscillations in the mixed state of the $\beta$ " salt is much more mysterious and poses the question on the peculiarity of this material.

\section{The basic equations and experiment}

In this section we consider a new mechanism for the quasiparticle scattering that goes beyond the usual 2D consideration of intralayer scattering by taking into account the interlayer-hopping contribution to the total scattering rate. This can explain an oscillationamplitude enhancement in the superconducting state for layered conductors with incoherent hopping across the layers.

In case the momentum across the layers is not preserved, the electron interlayer hopping maybe described in terms of an energy $\varepsilon$ that is distributed with the density of states (DOS) $g(\varepsilon)$. The energy spectrum of a layered conductor in a perpendicular magnetic field is, therefore, given by $E_{n}(\varepsilon)=\hbar \Omega(n+1 / 2)+\varepsilon$ [8]. The total DOS then follows from the standard Green-function definition 


$$
N(E)=\frac{1}{2 \pi^{2} l^{2}} \operatorname{Im} \sum_{n=0}^{\infty} \int d \varepsilon \frac{g(\varepsilon)}{E-E_{n}-\varepsilon-\Sigma_{n}(E)},
$$

where $\Sigma_{n}(E)$ is the average self energy corresponding to the $n$th Landau level and $l=(\hbar e / c B)^{1 / 2}$ is the magnetic length. For large energies and large $n \approx E / \hbar \Omega \gg 1$ (relevant for the magnetic quantum oscillations here) the self energy is independent on the index $n$ and the summation in Eq. (1) by use of Poisson's formula yields

$$
\frac{N(E)}{N(0)}=1+2 \operatorname{Re} \sum_{p=1}^{\infty}(-1)^{p} R_{D}(p, E) I_{p} \exp \left(\frac{2 \pi i p E}{\hbar \Omega}\right),
$$

where $N(0)$ is the $2 \mathrm{D}$ electron-gas DOS and the function $R_{D}(p, E)=\exp (-2 \pi p|\operatorname{Im} \Sigma(E)| / \hbar \Omega)$ generalizes the Dingle factor to the case of the energy-dependent self energy $\Sigma(E)$. The layer-stacking factor in Eq. (2),

$$
I_{p}=\int d \varepsilon g(\varepsilon) \exp \left(\frac{2 \pi i p \varepsilon}{\hbar \Omega}\right),
$$

is an important factor in the theory of magnetic quantum oscillations in normal and superconducting layered systems $[7,18]$. It describes contributions to the oscillations coming from the interlayer hopping. If the stacking is irregular $I_{p}$ becomes complex and contains a Dingle-like exponent [8].

The inverse scattering time $1 / \tau(E)=|\operatorname{Im} \Sigma(E)| / \hbar$ in the self-consistent Born approximation (SCBA) was found to be proportional to the total DOS [19-21]. Accordingly, the relation $N(E) / N(0)=$ $=\tau_{0} / \tau(E)$ holds, where $\tau_{0}$ is the intralayer scattering time $[22,23]$. Substituting this into Eq. (2) leads to an equation for $\tau(E)$ showing that it oscillates as a function of $1 / B$.

In case the highly anisotropic electronic system has a 3D Fermi surface the DOS related to the interlayer hopping is symmetric, $g(\varepsilon)=g(-\varepsilon)$, and becomes for nearest-neighbor hopping $g(\varepsilon)=\pi^{-1}\left(4 t^{2}-\varepsilon^{2}\right)^{-1 / 2}$. The corresponding layer-stacking factor then is given by $I_{p}=J_{0}(4 \pi t p / \hbar \Omega)$ [8]. This Bessel function oscillates as a function of $1 / B$ which is just another way to describe the well-known bottle-neck and belly oscillations of a corrugated 3D FS. Oscillating corrections to the Ginzburg - Landau expansion coefficients caused by the factor $J_{0}(4 \pi t p / \hbar \Omega)$ were also calculated in [24].

For the incoherent case, on the other hand, the translation invariance across the layers is lost. The irregular interlayer hopping means that the DOS deviates from the function $g(\varepsilon)=\pi^{-1}\left(4 t^{2}-\varepsilon^{2}\right)^{-1 / 2}$ and loses the symmetry $g(\varepsilon)=g(-\varepsilon)$ which implies that $\operatorname{Im} I_{p} \neq 0$. As will be shown below, this results in a special contribution to the electron scattering time which can be negative below $B_{c 2}$ and acts against the known intralayer damping mechanisms of the quantum oscillations in the mixed state [2]. However, this contribution vanishes if the hopping between the layers preserves the interlayer momentum leading to a corrugated 3D FS cylinder. This is an important point in our consideration.

Using Eq. (2) and $N(E) / N(0)=\tau_{0} / \tau(E)$ we write $\tau(E)^{-1}$ as a sum of the coherent (symmetric) and incoherent (asymmetric) terms:

$$
\tau(E)^{-1}=\tau(E)_{s}^{-1}-\tau(E)_{a}^{-1},
$$

$$
\begin{gathered}
\frac{\tau_{0}}{\tau_{s}}=1+2 \sum_{p=1}^{\infty}(-1)^{p} R_{D}(p, E) \operatorname{Re} I_{p} \cos \left(\frac{2 \pi p E}{\hbar \Omega}\right), \\
\frac{\tau_{0}}{\tau_{a}}=2 \sum_{p=1}^{\infty}(-1)^{p} R_{D}(p, E) \operatorname{Im} I_{p} \sin \left(\frac{2 \pi p E}{\hbar \Omega}\right) .
\end{gathered}
$$

With the help of the summation rule

$$
S(\lambda, \delta)=\sum_{p=-\infty}^{\infty}(-1)^{p} \mathrm{e}^{-|p| \lambda} \cos p \delta=\frac{\sinh \lambda}{\cosh \lambda+\cos \delta}
$$

one can rewrite Eqs. (5) and (6) in the integral form

$$
\frac{1}{\tau_{s(a)}}=\frac{1}{\tau_{0}} \int d \varepsilon g_{S(a)}(\varepsilon) S[\lambda, \delta(E, \varepsilon)] .
$$

Here $g_{s}(\varepsilon)=g_{s}(-\varepsilon)$ is the symmetric and $g_{a}(\varepsilon)=-g_{a}(-\varepsilon)$ is the antisymmetric part of the DOS $g(\varepsilon), \lambda(E)=2 \pi / \Omega \tau$, and $\delta(E, \varepsilon)=2 \pi(E-\varepsilon) / \hbar \Omega$. The SCBA, as well as Eqs. (4)-(8), are valid not only for point-like impurities but also for a smooth random potential provided its correlation radius is less than the Larmor radius, which holds for large $n$ [21]. One can see from Eqs. (4)-(8) that, in general, the incoherent contribution, $-\tau(E)_{a}^{-1}$, to the total scattering rate, $\tau(E)^{-1}$, is essential. The integral equation for $\tau(E)^{-1}$ is very complex and can be solved only perturbatively in the case $\lambda \gg 1$. In the limit $\lambda \rightarrow \infty$, when $S(\lambda, \delta) \rightarrow 1$, we have $\tau_{s}^{-1}=\tau_{0}^{-1}$ and $\tau_{a}^{-1}=0$. For finite, but large $\lambda$ the parameter $R_{D}(p, E)=\mathrm{e}^{-p \lambda} \ll<$. Even if $\Omega \tau \simeq 1$, the quantity $\mathrm{e}^{-\lambda}<<1$ and Eqs. (5) and (6) are just a series expansion in powers of the small parameter $\mathrm{e}^{-\lambda}$. Equation (7) shows the convergence of this series for any $\lambda>0$ allowing a perturbative solution. The perturbative terms oscillate as a function of $E$ and can be written as the series $\tau^{-1}(E)=\tau_{0}^{-1}\left[1+X_{1}+X_{2}+\right.$ $\left.+O\left(\mathrm{e}^{-3 \lambda_{0}}\right)\right]$, with $X_{1} \propto \mathrm{e}^{-\lambda_{0}}, \quad X_{2} \propto \mathrm{e}^{-2 \lambda_{0}}$, and $\lambda_{0}=2 \pi / \Omega \tau_{0}$. The first nonzero correction averaged 
over an oscillation period is proportional to $\overline{X_{1}+X_{2}}=-2 \lambda_{0} \mathrm{e}^{-2 \lambda_{0}}\left|I_{1}\right|^{2}$ and yields

$$
\frac{1}{\bar{\tau}}=\frac{1}{\tau_{0}}\left[1-\frac{4 \pi}{\Omega \tau_{0}}\left(R_{D}^{0}\right)^{2}\left(\operatorname{Re} I_{1}^{2}+\operatorname{Im} I_{1}^{2}\right)\right] .
$$

The Dingle factor $R_{D}^{0}=\exp \left(-2 \pi / \Omega \tau_{0}\right)$ is a small parameter in our perturbative solution. The term Im $I_{1}^{2}$ in Eq. (9) appears due to the incoherence.

It was established that in the $\beta^{\prime \prime}$ salt electron hopping across the layers is most probably incoherent, i.e., the momentum perpendicular to the layers is not preserved and there is no 3D Fermi surface [10]. The reason for this remarkable feature is unknown so far. It might be that some kind of disorder, such as different spatial configurations in the extraordinary large and complex anion-molecule layer may induce random hopping integrals, in analogy with intercalated layered compounds [8]. Furthermore, the $\beta^{\prime \prime}$ salt is the only material so far studied (not only among the BEDT-TTF salts) which displays an enhancement of the magnetic quantum-oscillation amplitude in the superconducting state [3].

This important result is summarized in Fig. 1. In this Dingle plot for a 2D metal the amplitudes $A_{1}$ of the fundamental dHvA frequency $(F \approx 198 \mathrm{~T})$ and $A_{2}$ of the second harmonic $(2 F)$ are normalized by $B \sinh (X) T^{-1}$ and plotted on a logarithmic scale as a function of $1 / B$, with $X=2 \pi^{2} k_{B} m_{c} T / e \hbar B$ and $m_{c}$ the effective cyclotron mass (see [3] for more details). Upon entering the superconducting state the oscillation amplitude $A_{1}$ is enhanced compared to the normal-state dependence (solid lines). For the second harmonic $A_{2}$ neither an additional damping nor an enhancement is observed.

In the superconducting state long-range order across the layers evolves through the renormalization

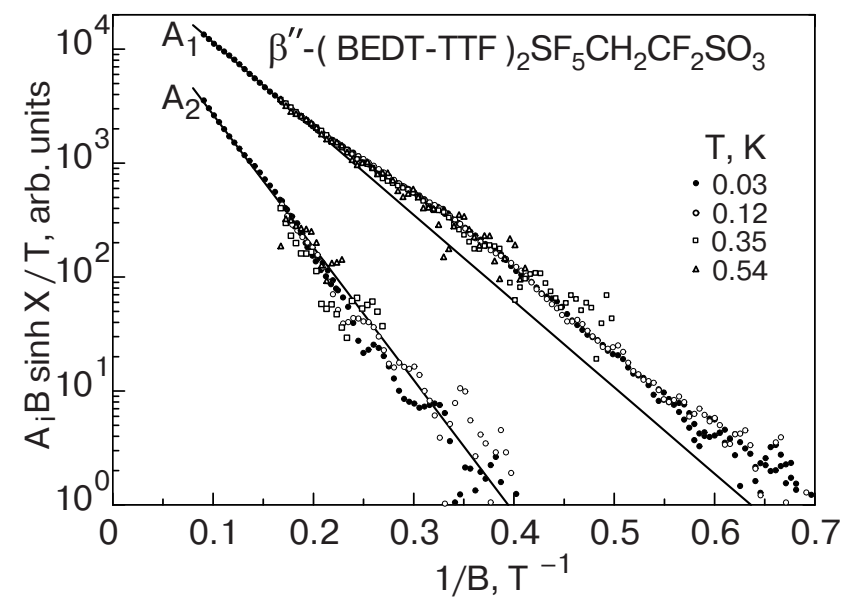

Fig. 1. Dingle plot of the fundamental $\left(A_{1}\right)$ and the second harmonic $\left(A_{2}\right)$ dHvA amplitudes of the $\beta^{\prime \prime}$ salt extracted from modulation-field data for different temperatures. The solid lines are fits to the data above $6 \mathrm{~T}$. of the hopping integrals [14]. This can be understood as follows. The quasiparticle hopping between the layers is an independent degree of freedom with respect to the in-plane Landau quantization. In the normal state the Green-function equation related to the interlayer hopping is given by

$$
\sum_{m}\left[\left(\varepsilon-\varepsilon_{i}\right) \delta_{i m}-t_{i m}\right] G_{i j}^{0}(\varepsilon)=\delta_{i j}
$$

where the electron energy in the layer $\varepsilon_{i}$ and the hopping integrals $t_{i m}=t_{i}\left(\delta_{i, m+1}+\delta_{i, m-1}\right)$ are assumed to depend on the layer indices for the sake of generality. In the superconducting state the order parameters in the layers, $\Delta_{i}$, become nonzero and the Gor'kov equation for the Green functions $G_{i j}$ can be written as

$$
\begin{gathered}
\sum_{m}\left[\left(\varepsilon-\varepsilon_{i}\right) \delta_{i m}-\hat{t}_{i m}\right] G_{i j}(\varepsilon)=\delta_{i j}, \\
\hat{t}_{i m}=t_{i m}-\Delta_{i} G_{i m}^{0}(-\varepsilon) \Delta_{m}^{*} .
\end{gathered}
$$

The star means the complex conjugate. When comparing Eq. (10) with Eq. (11) it is seen that in the superconducting state the effective hopping integrals, given by Eq. (12), become nonzero not only for next-nearest-neighbor hopping. In that case the nonvanishing retarded Green-function components $G_{i m}^{0}(-\varepsilon)$ result in nonzero $\hat{t}_{i m}$ for electron hopping between arbitrary sites $i$ and $m$. In fact, Eq. (12) simply reflects how the superconducting long-range order is established across the layers. In general the complex order parameters in the layers $\Delta_{i}=$ $=\left|\Delta_{i}\right| \exp (i \varphi)$ appear and result in interlayer (intrinsic) Josephson coupling [14]. In the absence of Josephson currents the order parameter can be chosen to be real and independent of the layer index $\Delta_{i}=\left|\Delta_{i}\right|=\Delta$. The correction to the DOS due to this mechanism is

$$
\delta g(\varepsilon)=-\Delta^{2} \sum_{i j} \frac{\partial g(\varepsilon)}{\partial t_{i j}} G_{i j}^{0}(-\varepsilon) .
$$

The second effect we have to take into account is caused by the vortex matter in the mixed state. Here, the vortices are disordered for fields slightly below $B_{c 2}$. They convert the degenerate Landau levels into asymmetric Landau bands as was shown in Refs. 25, 26. Thus, in the mixed state the quasiparticle tunneling between the layers implies the quantum transition between these Landau-band states which results in the additional contribution to the simple nearest-neighbor DOS

$$
\delta g_{\Delta}(\varepsilon)=\Delta^{2}\left(\frac{\partial g(\varepsilon)}{\partial \Delta^{2}}\right)_{\Delta=0} .
$$


The total correction to the DOS in the mixed state can be written as $\delta g_{\text {tot }}(\varepsilon)=\Delta^{2} G(\varepsilon)$, where $G(\varepsilon)$ is directly defined by Eqs. (13) and (14). Inserting $\delta g_{\text {tot }}(\varepsilon)$ into Eq. (8) and averaging over a period in $E$ results in

$$
\delta\left(\frac{1}{\tau}\right)=\frac{\Delta^{2}}{\tau_{0}} \int d \varepsilon G(\varepsilon) \overline{S(\lambda, \delta(E,-\varepsilon))}=\frac{\Delta^{2}}{\tau_{0}} \gamma .
$$

Since the DOS is normalized $\left(\int d \varepsilon g(\varepsilon)=1\right)$ the function $G(\varepsilon)$ satisfies the condition $\int d \varepsilon G(\varepsilon)=0$. This means that it is alternating in sign and $\gamma$ might be negative because $\overline{S(\lambda, \delta(E,-\varepsilon))}>0$. The studied system is too complex to calculate $\gamma$ in general. In the limit $\lambda \rightarrow \infty$ this coefficient vanishes since $\overline{S(\lambda, \delta(E,-\varepsilon))} \rightarrow 1$. It is instructive to consider a correction to the scattering rate in Eq. (9) in the mixed state. The variation of the layer-stacking factor $\delta I_{1}$ is given by Eq. (3) with the DOS replaced by $\delta g_{\text {tot }}(\varepsilon)$. The broadening of the Landau levels, caused by $\delta g_{\text {tot }}(\varepsilon)$, is of the order of the width of this function and much less then $\hbar \Omega$ in order to observe the oscillations. Therefore, in first approximation $\operatorname{Re} \delta I_{1} \approx 0$ and

$$
\operatorname{Im} \delta I_{1}=\Delta^{2} \int d \varepsilon G(\varepsilon) \sin \left(\frac{2 \pi \varepsilon}{\hbar \Omega}\right) \approx \Delta^{2}\left(\frac{2 \pi\langle\varepsilon\rangle}{\hbar \Omega}\right),
$$

where $\langle\varepsilon\rangle=\int d \varepsilon G(\varepsilon) \varepsilon$. (For $G(\varepsilon)=-G(-\varepsilon), \operatorname{Re} \delta I_{1}$ is zero exactly.) Thus, the correction to the scattering rate in the mixed state near $B_{c 2}$, caused by the interlayer-hopping mechanism, is given by

$$
\delta\left(\frac{1}{\bar{\tau}}\right)=-\Delta^{2} \frac{2 \operatorname{Im} I_{1}}{\tau_{0}}\left(\frac{4 \pi}{\Omega \tau_{0}}\right)\left(R_{D}^{0}\right)^{2}\left(\frac{2 \pi\langle\varepsilon\rangle}{\hbar \Omega}\right) .
$$

For $\langle\varepsilon\rangle \operatorname{Im} I_{1}>0$, this gives a decrease in the scattering rate. Note that the latter is nonzero only if the system is incoherent in the normal state and Im $I_{1} \neq 0$. This strongly supports the relevance of this mechanism for the $\beta "$ salt, since only this organic metal displays both incoherence in the normal state and an enhancement of the $\mathrm{SdH}$ and $\mathrm{dHvA}$ amplitudes in the mixed state.

Thus, the overall effect superconductivity has on $R_{s}$ is determined by the balance between positive and negative contributions to the scattering rate. The already mentioned positive contribution from the intralayer scattering at vortices and defects is

$$
\frac{\hbar}{\tau_{s c}}=\Delta^{2}\left[\left(\frac{\pi}{\mu \hbar \Omega}\right)^{1 / 2}+\frac{\beta}{\mu \Omega \tau_{0}}\right] .
$$

The new additional interlayer mechanism we discuss here results in a negative contribution given by $\delta(1 / \tau)=\left(\Delta^{2} / \tau_{0}\right) \gamma[$ Eq. (15)]. Since little is known about the DOS of the studied system, even in the normal state, we cannot calculate the coefficient $\gamma$ quantitatively. However, contrary to Eq. (17), for this term the small factor $1 / \mu$ is absent, so that the overall correction to the scattering rate might be negative. The experimental facts [3] give us confidence that this is the case at least for the $\beta$ " salt.

\section{Conclusions}

We conclude with a qualitative picture of the effect discussed here. The incoherence means that the hopping time between the layers $\tau_{z} \approx \hbar /|t| \gg>\tau_{0}$ so that an electron scatters many times within a layer before leaving it [11]. Here the quantity $|t|$ is some averaged hopping integral that in the $\beta$ " salt may be assumed to be the smallest parameter in energy. Indeed, experimentally $|t|$ cannot be resolved in the $\beta$ " salt reflecting the fact that the hopping integral is one of the smallest for all known 2D organic metals so far [10]. Consequently, even small spatial fluctuations of the hopping probability within and across the layers render the electron motion across the layers incoherent. On the other hand, for the evolution of superconductivity some interlayer (Josephson) coupling is vitally important. Long-range order is established below $B_{c 2}$, thereby renormalizing the hopping integral. According to Eq. (12), the renormalized $\tau_{z}$ in the superconducting state may be estimated as $\tau_{z} \approx \hbar / t+\Delta^{2} / t \mid$. For $\Delta \gg|t|$ the hopping time reduces considerably and becomes $\tau_{z} \approx \hbar|t| / \Delta^{2}$. The latter means that the quasiparticles spend less time within the (impurity-containing) layers decreasing the scattering rate and, consequently, enhancing the Dingle factor. In the $\beta "$ salt this effect is strong because of the smallness of $|t|$. Thus, our mechanism relates the two unusual effects observed in the $\beta$ " salt: the incoherent interlayer hopping transport and the enhancement in the quantum-oscillation amplitudes in the mixed state.

One final remark is in order. Qualitatively, in the normal state the conductivity is proportional to the scattering time of electrons on impurities. A transition into the superconducting state means that new quasiparticles (the Cooper pairs) do not scatter on impurities and the scattering time became infinite. In the incoherent quasi-2D conductors there are two contributions to the Dingle factor. The intralayer scattering on the Abrikosov vortices decreases the scattering time and, correspondingly, the Dingle factor. Contrarily, the interlayer scattering time in the incoherent layered conductors increases in the superconducting state because quasiparticles move more freely across the layers when the long-range-order establishes in this di- 
rection. The corresponding effect on the Dingle factor is its enhancement. If this enhancement is larger than decrease due to the intralayer scattering, the total effect of the superconductivity on the $\mathrm{SdH}$ and $\mathrm{dHvA}$ oscillations would be an increase of their amplitudes since both $\mathrm{SdH}$ and dHvA oscillations depend on the Dingle factor. Therefore, the long-range-order across the layers decreases the interlayer scattering rate in the superconducting state, restores the coherence, and decreases the Dingle factor. In coherent layered conductors with the 3D FS the momentum across the layers is preserved which assumes no scattering in the interlayer electron hopping. Thus, the simple and «counterintuitive» physics we discuss here works only if in the normal state electron hopping is incoherent. All theories of the quantum magnetic oscillations in layered superconductors so far have ignored the interlayer hopping and have formed a wrong intuition that oscillation amplitudes should always be decreased in the superconducting state. We have shown in this paper that this may be not the case in the incoherent layered conductors like the $\beta "$ salt. The problem is complex and further experiments with layered organic conductors and/or artificially created superlattices are necessary to check the ideas of our paper.

This work was supported in part by INTAS, project INTAS-01-0791, and the NATO Collaborative Linkage Grant No. 977292. V.M.G. thanks P. Fulde and S. Flach for the hospitality at the MPIPKS in Dresden and P. Wyder, I. Vagner, T. Maniv, P. Grigoriev, A. Dyugaev, M. Kartsovnik, and W. Biberacher for useful discussions.

1. J.E. Graebner and M. Robbins, Phys. Rev. Lett. 36, 422 ( 1976).

2. T. Maniv, V. Zhuravlev, I. Vagner, and P. Wyder, Rev. Mod. Phys. 73, 867 (2001).

3. J. Wosnitza, J. Hagel, P.J. Meeson, D. Bintley, J.A. Schlueter, J. Mohtasham, R.W. Winter, and G.L. Gard, Phys. Rev. B67, 060504(R) (2003).

4. K. Maki, Phys. Rev. B44, 2861 (1991).

5. M.J. Stephen, Phys. Rev. B45, 5481 (1992).

6. A. Wasserman and M. Springford, Physica B194-196, 1801 (1994)

7. V.M. Gvozdikov and M.V. Gvozdikova, Phys. Rev. B58, 8716 (1998).
8. V.M. Gvozdikov, Sov. Phys. Solid State 26, 1560 (1984); ibid. 28, 179 (1986); Fiz. Nizk. Temp. 12, 705 (1986) [Sov. J. Low Temp. Phys. 12, 399 (1986)].

9. V.M. Gvozdikov, Phys. Rev. B70, 085113 (2004).

10. J. Wosnitza, J. Hagel, J.S. Qualls, J.S. Brooks, E. Balthes, D. Schweitzer, J.A. Schlueter, U. Geiser, J. Mohtasham, R.W. Winter, and G.L. Gard, Phys. Rev. B65, 180506(R) (2002).

11. R.H. McKenzie and P. Moses, Phys. Rev. Lett. 81, 4492 (1998); P. Moses and R.H. McKenzie, Phys. Rev. B60, 7998 (1999).

12. J. Wosnitza, Fermi Surfaces of Low-Dimensional Organic Metals and Superconductors, Springer, Berlin (1996); J. Singleton, Rep. Prog. Phys. 63, 1111 (2000); M.V. Kartsovnik and V.G. Peschansky, Fiz. Nizk. Temp. 31, 249 (2005).

13. I.M. Lifshitz and A.M. Kosevich, Zh. Eksp. Teor. Fiz. 29, 730 (1956).

14. V.M. Gvozdikov, Fiz. Nizk. Temp. 14, 15 (1988) [Sov. J. Low Temp. Phys. 14, 7 (1988)].

15. G. Goll, M. Heinecke, A.G.M. Jansen, W. Joss, L. Nguen, E. Steep, K. Winzer, and P. Wyder, Phys. Rev. B53, 8871 (1996).

16. T. Terashima, C. Haworth, H. Takeya, S. Uji, and H. Aoki, Phys. Rev. B56, 5120 (1997).

17. L.P. Gor'kov and J.P. Schrieffer, Phys. Rev. Lett. 80, 3360 (1998).

18. V.M. Gvozdikov, A.G.M. Jansen, D.A. Pesin, I.D. Vagner, and P. Wyder, Phys. Rev. B70, 245114 (2004); ibid. 68, 155107 (2003); V.M. Gvozdikov, Yu.V. Pershin, E. Steep, A.G.M. Jansen, and P. Wyder, Phys. Rev. B65, 165102 (2002); V.M. Gvozdikov, Fiz. Nizk. Temp. 27, 956 (2001) [Low Temp. Phys. 27, 704 (2001)].

19. T. Ando and Y. Uemura, J. Phys. Soc. Jpn. 36, 959 (1974).

20. T. Maniv and I.D. Vagner, Phys. Rev. B38, 6301 (1988).

21. M.E. Raikh and T.V. Shahbazyan, Phys. Rev. B47, 1522 (1993).

22. P.D. Grigoriev, Phys. Rev. B67, 144401 (2003).

23. T. Champel and V.P. Mineev, Phys. Rev. B66, 195111 (2002).

24. T. Champel and V.P. Mineev, Philos. Mag. B81, 55 (2001).

25. M.R. Norman and A.H. MacDonald, Phys. Rev. B54, 4239 (1996).

26. Z. Tesanovich and P.D. Sacramento, Phys. Rev. Lett. 80, 1521 (1998). 